\title{
DE LA TRADICIÓN GÓTICA EN LA LITERATURA HISPANOAMERICANA: «LA GRANJA BLANCA», DE CLEMENTE PALMA
}

\author{
ENCARNACIÓN LÓPEZ GONZÁLVEZ \\ Universidad de Guadalajara (México) \\ elgonzalvez@gmail.com
}

Recibido: 15-06-2014

Aceptado: 19-11-2014

(C) (1)

\section{RESUMEN}

En el presente trabajo se ejemplifica la presencia de la tradición gótica, a partir de lo que se ha denominado American Gothic, en el texto "La granja blanca», de Clemente Palma. Para ello, se señalan algunos textos y temas ampliamente vinculados e identificados con dicha tradición, como los del escritor estadounidense Edgar A. Poe «El retrato oval», «Ligeia» o «Morella»; y se apuntan brevemente otras características del gótico literario, como es el caso del exceso y la transgresión, en palabras de Fred Botting (1996) o la transformación de los personajes femeninos en referencia precisa al American Gothic, como señala Louis S. Gross (1989). Todo lo anterior con el fin de rastrear una de las huellas y presencia de la tradición gótica de corte anglosajón en la literatura hispanoamericana.

Palabras Clave: Literatura gótica, literatura hispanoamericana, modernismo, American Gothic, transgresión.

\section{Abstract}

This work illustrates the presence of what is known as the "American Gothic» in Clemente Palma's text «La granja blanca». To this end, several texts and themes related with this tradition are notes, such as Edgar Allan Poe's «Oval Portrait», «Ligeia» or «Morella»; other internal and external features of literary gothicism are also pointedout, such as excess and transgression (Fred Botting: 1996) or, referring to the inner characteristics of the female gender, its transformation in an explicit reference to American Gothic (Louis S. Gross: 1989). In light of the aforementioned, we will seek the traces and presence of the Anglosaxon Gothic tradition in Hispanic Literature. 
Keywords: Gothic Literature, Latin American Literature, Modernism, American Gothic, transgression.

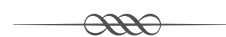

Clemente Palma (1872-1946) es un autor poco conocido en la actualidad, su obra no es extensa y tampoco fácil de conseguir, ha pasado a formar parte de la lista de autores extraños y casi olvidados. Publicó dos libros de cuentos, Cuentos malévolos (1904) e Historietas malignas (alrededor de 1920), y una novela de ciencia ficción, XYZ (1934), basada, según el propio autor, en la idea que desarrolló Villiers d l'Isle Adam en la Eva futura, aunque adecuada al ámbito del cine.

Sus textos y la temática de los mismos han sido vinculados con frecuencia con la tradición fantástica, de ciencia ficción y a veces con la gótica, sobre todo en lo que concierne a lo extraño e inquietante; en ellos es frecuente encontrar temas como la necrofilia, el incesto y el ocultismo. Esto nos lleva a afirmar, como ha apuntado Gabriela Mora (2000), ${ }_{1}^{1}$ que Palma podría conectarse con cierta tradición de la literatura gótica, puesto que en sus textos nos hallamos frente a frente con lo perturbador que, en gran medida, ha caracterizado a la literatura gótica desde su inicio. No es intención de este trabajo argumentar concienzudamente si podría considerarse a Palma como un escritor gótico o no, sobre todo por cuestión de tiempo y espacio; sin embargo sí me gustaría señalar cómo el cuento «La granja blanca» puede conectarse con la tradición gótica por varios motivos: en primer lugar, por la presencia de temas y características propias del género, y en segundo lugar por la presencia de ciertos textos que sí pertenecen sin lugar a dudas al mismo, como es el caso de «Morella» y «Ligeia» de Edgar Allan Poe.

En El cuento peruano hasta 1919, Ricardo González Vigil señala al respecto de la labor narrativa de Clemente Palma, que pese a que considera que está sobrevalorado -menciona para ello los testimonios de Luis Alberto Sánchez o de Alberto Escobar, autores que lo ubican como uno de los mejores cultivadores de lo fantástico en Perú y América-, apunta que hay dos momentos diferentes en la narrativa de Palma que deben tenerse en cuenta. Por un lado, la publicación de obras de corte realista con el pseudónimo Juan Apapu-

1 Véase Gabriela Mora (2000) o el artículo en el que esboza un vínculo similar (1996: 62-69). 
cio Corrales, cuya labor destaca principalmente como renovadora en ciertos aspectos de la narración costumbrista. Y, por otro lado, la obra que publicó con nombre propio de índole fantástica, sobre todo a partir de Cuentos malévolos, «y que lo muestra embriagado de decadentismo, truculencia, voluptuosidad, agnosticismo y tono «maldito» (aprendido más que en los franceses en los maestros rusos: el humor negro de Andreiev, Gogol y Averchevko)» (González Vigil, 1992: 640).

Fred Botting (1996: 1-20) señala dos características principales de la novela gótica inaugurada por Walpole en 1767: el exceso y la transgresión. El exceso, implícitamente transgresor, viene referido a cruzar o traspasar los límites de la realidad y lo posible, así como de la moralidad, mostrando de este modo las pasiones más descarnadas y las conductas más depravadas, representadas la mayoría de las veces en la figura del villano. Por otro lado, el exceso gótico, referido en la estética gótica a los sentimientos y las emociones reflejadas formalmente durante el primer gótico en un exceso de ornamentación, se refiere a la ausencia de límites de la novela gótica en este sentido, lo cual formaba parte de cierta reivindicación estilística frente a la novela del periodo neoclásico de corte realista, que insistía en las reglas estéticas de claridad, mesura y equilibrio. Explica Botting:

In Gothic productions imagination and emotional effects exceed reason. Passion, excitement and sensation transgress social properties and moral laws [...]. Drawing on the myths, legends and folklore of medieval romances, Gothic conjured up magical worlds [...]. Associated with wildness, Gothic signified an over-abundance of imaginative frenzy, untamed by reason and unrestrained by conventional eighteenth-century demands for simplicity, realism or probability. The boundlessness as well as the over-ornamentation of Gothic styles were part of a move away from strictly Neoclassical aesthetic rules [...] (1996: 3)

De este modo, el exceso es implícitamente transgresor puesto que en su uso de la sobre-ornamentación y la ausencia de límites traspasa continuamente las barreras de lo socialmente «normal». Por ello, la novela gótica se puede considerar esencialmente transgresora, ya que cuenta lo que nadie quiere o se atreve a contar, los temores más profundos del ser humano, moviéndose en un ambiente oscuro y de temor y sin atenerse a ningún tipo de límites sociales. Paradójicamente toda esta transgresión sirve para restablecer y fortificar ciertos valores sociales:

The terrors and horrors of transgression in Gothic writing become a powerful means to reassert the values of society, virtue and property: transgression, by 
crossing the social and aesthetic limits, serves to reinforce or underline their value and necessity, restoring or defining limits [...]. The tortuous tales of vice, corruption and depravity are sensational examples of what happens when the rules of social behaviour are neglected. (1996: 7)

Así, no sería desatinado llegar a afirmar que la novela gótica llega a ser, en este sentido, un género tradicionalmente conservador, aun cuando existen textos que precisamente por no restablecer el orden -como The Vampyre de Polidori, «The True Story of a Vampire» de Stenbock, Frankenstein de Mary Shelley, por ejemplo-, abren la posibilidad de un cuestionamiento de los valores sociales y una necesidad de cambio, más que de reafirmación de lo establecido. Sin embargo, a pesar de este «aspecto» conservador no podemos olvidar que un género de esencia transgresora siempre representa un desafío y conlleva a cierto cuestionamiento, aun cuando los límites vuelvan a restablecerse y a reafirmarse lo que se había puesto en duda.

El modo de representación de la transgresión en la novela gótica fue cambiando desde una exteriorización de lo gótico, sobre todo durante el siglo xVIII, dada por la ambientación espacial, esto es, castillos, ruinas, galerías, bosques tenebrosos..., que provoca terror; hasta una interiorización psicológica que se da fundamentalmente a partir del siglo XIX, basada en los ideales románticos de individualidad y que provoca, de este modo, horror. Ya no son necesarias las oscuras galerías arquitectónicas, pues estas se encuentran en los recovecos de la mente. En este proceso de interiorización, la concepción del sentimiento de lo sublime como algo que despierta el temor adquirirá mucha importancia, tal como veremos más adelante.

Debido a este proceso de interiorización del gótico, influencia de las ideas románticas de individualidad, la novela gótica desemboca en lo que se ha denominado «gótico psicológico» o romántico, sobre todo a partir de 1820. En este, el espacio se convierte en psicológico, «convirtiendo el secuestro en mental, además de la detención física, con personajes atrapados por mentes, ciudades, familias y estructuras sociales obsesionadas» (Solaz, 2003). A partir de este momento el género establece dos caminos principales: seguir los cánones establecidos por Walpole, o no hacerlo, como fue el caso del American Gothic o "gótico americano o sureño», ${ }^{2}$ que exploró y criticó la cultura del sur de Estados Unidos basándose en aspectos como la raza, el género, la religión y la política a partir de un cuestionamiento de la lógica, la moralidad y la humanidad.

2 Nos referiremos con "gótico americano", siguiendo una traducción literal del término, al gótico exclusivo de Estados Unidos. 
Otra de las características internas del género gótico que establece Botting se refiere a lo sublime. Tal como ya habíamos mencionado, la novela gótica ofrece una apuesta estética basada en los sentimientos y las emociones, radicalmente opuesta a los cánones neoclásicos basados en la armonía y la mesura. Así, no es de extrañar que esta «libertad» desemboque durante el romanticismo en el sentimiento de lo sublime. Por ello, y debido al proceso de interiorización o subjetivización ya apuntado más arriba, la sublimidad de los paisajes se convierte en una simple marca del proceso interno de pensamiento o ánimo de los personajes. Fred Botting define lo sublime para los románticos como lo que «stimulated powerful emotions of terror and wonder in the viewer. Their immense scale offered a glimpse of infinity and awful power, intimations of a metaphysical force beyond rational knowledge and human comprehension [...]. Linked to poetic and visionary power, the sublime also evoked excessive emotions» (1996: 4).

Lo sublime, entonces, sería el sentimiento que se despierta en el ser humano ante la contemplación o experimentación de ciertas situaciones o emociones, como el miedo, cuya cercanía a la sensación de infinitud provoca la expansión espiritual y anímica y, consecuentemente, la reflexión metafísica: «Wonder, awe, horror and joy were the emotions believed to expand or elevate the soul and the imagination with a sense of power and infinity. Mountains were the foremost objects of the natural sublime» (1996: 38).

Por otro lado, se considera que la primera novela del gótico americano fue Wieland (1796), de Charles Brockden Brown. El texto inauguró un género que, a pesar de guardar reminiscencias del británico, pronto se adaptó a la nueva sociedad americana y generó algunas características propias. En este sentido, el gótico permitió a los autores americanos, al igual que a los británicos, explorar nuevos horizontes de la fantasía, lo cual ofreció resultados diferentes.

Una de las primeras consideraciones importantes que hay que tener en cuenta a la hora de abordar las especificidades del género en Estados Unidos es que los primeros pobladores europeos que llegaron en el siglo XVII fueron los puritanos provenientes de las corrientes más conservadoras del protestantismo, quienes posiblemente vieron la tierra como un Nuevo Mundo, un paisaje vasto e imponente. Sobre ellos recayó la construcción de una nueva sociedad, la sociedad americana, que fue creada a partir de ciertos preceptos basados en el temor de Dios, la culpabilidad heredada del pecado original y la visión de la mujer como responsable de este.

En el planteamiento de los nuevos pobladores hay una dicotomía que combina lo sagrado y lo profano; estos pobladores eminentemente religiosos 
y moralistas reencarnan el deseo burgués de hacerse a sí mismos, por su condición de migrantes en una tierra nueva y por las posibilidades de novedad que el territorio americano ofrecía, y hacerlo bajo los preceptos de lo moralmente correcto que Dios impone. Louis S. Gross señala que este planteamiento de vida entre lo profano y lo sagrado fue un aspecto decisivo que caracterizó la naturaleza de la sociedad americana y está presente en Wieland (1989: 6-7).

Wieland, aun cuando es la iniciadora del gótico en Estados Unidos, posee ciertas características que se mantendrán a lo largo del desarrollo del género. Tal es el caso de los personajes a caballo entre Europa y América. La familia Wieland proviene de Europa y la novela narra su experiencia en tierra americana. Esta situación servirá para el contraste continuo entre el Nuevo y el Viejo Continente y la nueva vida que se abre alejada de este último.

También es un punto destacable la importancia de la soledad para la definición e identidad del individuo. Los personajes enfrentados a sí mismos y a sus miedos provocan un cambio que es al mismo tiempo temido y deseado porque supone, como afirma Gross, el temor a la transgresión de los límites sexuales y morales. Este cambio frecuentemente se exterioriza con transformaciones en el cuerpo del personaje o en la actitud del mismo.

En Wieland aparece otro rasgo que será característico para el género americano y es el relacionado con la voz narrativa. El narrador de Wieland está teñido por su propia subjetividad y es además actor, haciendo de su psicología la desencadenante del terror. Dicho narrador homodiegético o en primera persona gramatical, generalmente forma parte de una familia atípica, mediante la cual ha obtenido su educación y la que suele acentuar el sentimiento de soledad, represión y consecuentemente temor. Muchas veces es, por tanto, este tipo de familia la que provoca el cambio o metamorfosis del protagonista. A esta voz narrativa hay que añadir que la acción narrativa tiene lugar en un presente al que pertenece el narrador/actor.

Eric Savoy señala la alegoría como una de las características del gótico americano (1998: 3-19); mediante la alegoría cobran importancia ciertos aspectos que en apariencia son menos relevantes del relato, como es el caso de la casa o los paisajes, elementos que llegan a convertirse en claves a la hora de provocar el sentimiento de miedo. En este proceso de lectura alegórica la prosopopeya se hace especialmente interesante ya que a partir de ella elementos como la casa encarnan roles personificados y psicológicos muy específicos, aun cuando en muchas ocasiones se pueden leer como ampliaciones de la propia psique del narrador/actor, motor fundamental del miedo del personaje 
y al mismo tiempo del lector, debido a la identificación de este con aquel. Un ejemplo claro lo encontramos en «La caída de la casa de Usher», de Edgar Allan Poe.

A estas características Gross añade la de las «mujeres que se transforman» para referirse a ciertos personajes femeninos del gótico americano, construidos como una especie de «mujeres oscuras» o asociadas culturalmente al mal (como Lilith, Cleopatra u otros personajes arquetípicos similares), cuya malignidad se construye a partir de la transformación, que les permite demostrar la fuerza de su poder y voluntad. Tal es el caso de la Ligeia o la Morella de Poe (1989: 37-53).

Hasta aquí hemos mencionado algunas de las características principales que distintos estudios literarios han considerado como propias o incluso constitutivas del gótico americano. En adelante, veremos si están presentes o no en el cuento «La granja blanca», y de qué manera lo hacen, pero antes de ello sería importante apuntar el papel fundamental de los escritos de Poe en la literatura fantástica de los textos de final del siglo XIX.

En este sentido, hay que destacar la difusión y presencia de algunas traducciones de los textos de Poe en revistas literarias de la época. ${ }^{3}$ Sus escritos, así como el peculiar enfoque y tratamiento del hecho extraño, fueron de importancia decisiva para dar un sesgo diferente a la literatura fantástica en español, así como para renovar temas y modos narrativos que se consideraban anquilosados para el final del siglo xix. Por medio de Poe, podría afirmarse casi sin reservas que la «nueva» estética decadentista se introdujo de manera importante en la literatura en español, y la ambigüedad del texto, rasgo casi esencial en el gótico americano, como se vio más arriba, permeó en cierto sentido los textos de la tradición hispanoamericana, desde las tramas argumentales de resolución ambigua hasta personajes que se mueven a caballo entre lo tangible y lo ilusorio.

En «La granja blanca», de Palma, es evidente esta presencia de la literatura de Poe desde el mismo argumento del cuento, que recuerda a la vez al de «Morella» o «Ligeia». A este respecto, Gabriela Mora señala que el cuento de Palma y los dos de Poe comparten el narrador homodiegético y el retorno de ultratumba (Mora, 1996: 63).

«La granja blanca» cuenta a partir de un narrador en primera persona gramatical la historia del protagonista (del que desconocemos el nombre) y su prima (novia, prometida y esposa del mismo), Cordelia, quien muere, regresa

3 Para más información, véase el trabajo de Hensley C. Woodbridge (1969: 18-19). 
de la tumba y deja como prueba irrefutable de su retorno una hija, con la que el protagonista pretende casarse, una vez haya crecido; por esta razón el maestro del protagonista, uno de los pocos personajes secundarios del cuento, interviene y arroja a la niña por la ventana para matarla y frustrar de esta manera los planes del enloquecido protagonista. El cuento finaliza con reminiscencias de «El horla» de Maupassant, ya que ambos protagonistas prenden fuego al lugar (con los sirvientes dentro) en el que han perdido la cordura, mostrando de este modo la pérdida total del juicio, al menos moral.

Es innegable que Palma reconstruye el argumento de Poe, a su vez un argumento tratado desde la antigüedad, el regreso de la tumba, y le da un giro de tuerca: en «La granja blanca» el protagonista no es solo consciente de que Cordelia se ha reencarnado en la hija, cosa que sí ocurre en «Morella». El protagonista de Morella quiere darle voluntad propia a la hija, para que deje de ser la madre, a la que teme y en el fondo odia; en cambio, Palma decide anular cualquier personalidad propia de la hija para que sea de nuevo la madre y pueda casarse con ella; así, el exceso viene implícito en un argumento que propone, primero, el regreso de la tumba y luego el incesto como solución moral al amor frustrado. Se rompen de esta forma los límites naturales de la realidad, cosa que plantea el autor desde el primer fragmento ( $¿$ Realmente se vive o la vida es una ilusión prolongada?», dice el narrador en la primera línea), y refleja la transgresión como componente esencial de la acción narrativa.

En los cuentos de Poe, el amor acaba estando en cierta forma intelectualizado, mientras que en «La granja blanca» parece que este es el motor de la acción narrativa, puesto que el único motivo que tiene Cordelia para regresar de la muerte no es la voluntad o los celos, como ocurre con Morella y Ligeia, respectivamente, sino el amor o incluso la idea romántica de enfrentarse al destino fatal.

Si recordamos lo apuntado más arriba, una de las características esenciales del gótico americano venía dada con la transformación del personaje que tiene lugar casi siempre a partir del encuentro vinculado con lo sobrenatural. Así, el narrador protagonista de «La granja blanca» es un sujeto normal hasta que es consciente de que Cordelia estuvo muerta y regresó a la vida, por un lado, y lo ve de forma tangible en la persona de Cordelia hija; en ese momento algo cambia y provoca la pérdida del juicio moral, esto es, lo que es o no correcto socialmente hablando: «Las incoherencias del aterrado maestro y una frase que exclamó: «iEs Cordelia que renace!», abrieron ante mis ojos un horizonte inmenso, terrible...». 
Otro de los aspectos señalados al principio se relacionaba con la coetanidad de los hechos, cosa que el texto también cumple en cuanto que es evidente que lo que narra ha tenido lugar al mismo tiempo en el que supuestamente vivió el narrador; no está ubicado en un pasado remoto, como otros textos del primer gótico británico.

El espacio es relevante también, entendido como alegoría y como proyección del estado anímico del protagonista. La granja blanca donde tiene lugar la vida idílica de esta pareja de amores ideales está ubicada a las afueras, alejada de la civilización; en un principio está en contacto con la naturaleza, rodeada de flores, espacios hermosos y limpios. Sin embargo, cuando el narrador sufre este cambio de juicio decide quemarla, aniquilarla, como se ha eliminado asimismo la ilusión, la esperanza y la razón del protagonista.

Si bien es cierto que Cordelia sufre una transformación que es la que sustenta, por un lado, la posible lectura sobrenatural del texto y, por otro, la pérdida de juicio moral del protagonista, lo cual provoca, a su vez, la repulsión moral del resto de la sociedad ante la idea del incesto, representada en el personaje secundario del maestro, esta transformación no está impregnada de atributos que connoten una naturaleza maligna, como podría ocurrir con el personaje de Morella. Por el contrario, Cordelia se construye como la bondad misma. Su belleza física se relaciona más con la estética prerrafaelita que con la de femme-fatale, lo cual la acerca incluso a la ingenuidad o la bondad. No hay en ello una muestra de amor ideal, sino por el contrario la pasión y el amor carnal es parte fundamental de esta relación, lo que la aleja por un lado de la irrealidad planteada en el amor intelectualizado de los personajes femeninos de Poe y, por otro, de esta idea de mujer ideal lejana a lo terrenal.

Uno de los motivos que provocan la desaparición definitiva de Cordelia es el retrato, lo cual tiene reminiscencia no solo de uno de los motivos largamente vinculados con la literatura de corte fantástico o sobrenatural, sino también con otro texto de Poe bien conocido: «El retrato oval». La diferencia principal podríamos apuntar que reside en que es ella misma la que se pinta y cuando desaparece también lo hace del cuadro, como ocurre también en otro texto de importancia para el tema como es El retrato de Dorian Gray. La transformación de Cordelia, esto es, su regreso de la tumba, estaba anunciada desde el comienzo del texto puesto que el narrador la compara con la doncella que aparece en La resurrección de la hija de Jairo, un cuadro que hay en la capital. Es obvia la relación entre ambos textos así como el indicio temático de la acción narrativa.

Asimismo, lo que preocupa realmente al autor, más allá de lo incestuoso o el tema del regreso de la muerte, es la cuestión ontológica de la realidad, 
lo que da pie a la justificación fantástica del argumento: «¿Realmente se vive o la vida es una ilusión prolongada? ¿Somos seres autónomos e independientes en nuestra existencia?» (Palma, 1974: 123).

Así, Cordelia logra dar vida después de muerta, conjugando de este modo la dicotomía vida/muerte de una manera efectiva, y la ilusión de ambas, precepto fundamental del que parte la narración.

«La granja blanca» constituye un relato que contiene, de forma evidente, un modo de narrar y entender lo aparentemente sobrenatural que se vincula con el gótico americano, tal como hemos visto: el uso del narrador actor y la ambigüedad que esto provoca, la coetaneidad de los hechos narrados, el espacio alegórico, la transformación del protagonista y la construcción de lo sobrenatural a partir de un personaje femenino cuya naturaleza cambiante transgrede los límites naturales y la realidad. Son todos ellos elementos que identifican «La granja blanca» como un cuento conformado a partir del gótico.

\section{BIBLIOGRAFÍA}

BotTING, Fred (1996): Gothic, Routledge, Londres.

GonZÁlez VigIL, Ricardo (1992): El cuento peruano hasta 1919, Cope-Petroperú, Lima.

Gross, Louis S. (1989): Redefining American Gothic: from Wieland to Day of the Dead, Ann Arbor, UMI Research Press (Studies in Speculative Fiction, 20).

MorA, Gabriela (1996): ««La granja blanca», de Clemente Palma: relaciones con el decadentismo y Edgar Allan Poe», Revista de la Casa de las Américas, núm. 205, pp. 62-69.

(2000): Clemente Palma: el modernismo en su versión decadente y gótica, Instituto de Estudios Peruanos (Lengua y sociedad, 16), Lima.

Palma, Clemente (1974): «La granja blanca», Cuentos malévolos, Peisa, Lima.

SAvor, Eric (1998): «The Face of the Tenant. A Theory of American Gothic», en Robert K. Martin y Eric Savoy (eds.), American Gothic: New Interventions in a National Narrative, University of Iowa Press, Iowa, pp. 3-19. < http://dx.doi.org/10.1017/ccol0521791243.009>

SolAz, Lucía (2003): «Literatura gótica», Espéculo. Revista de Estudios Literarios, disponible en 〈http://www.ucm.es/info/especulo/numero23/gotica.html [marzo de 2014].

Stevens, David (2000): The Gothic Tradition, Cambridge University Press (Cambridge Contexts in Literature), Cambridge.

Woodbridge, Hensley C. (1969): «Poe in Spanish America: A Bibliographical Supplement», Poe Newsletter, vol. II, núm. 1 (enero), pp. 18-19. Versión digitalizada disponible en http://www.eapoe.org/pstudies/ps1960/p1969105.htm). < http://dx.doi.org/10.1111/j.1754-6095.1969.tb00112.x> 